\title{
Diseño de una estratégia didáctica centrada en potenciar el pensamiento computacional de jóvenes con Enfermedades Raras de la asociación INèDITHOS
}

\author{
Sebastià Verger Gelabert ${ }^{1}$ \\ Marc Salas Colom ${ }^{2}$ \\ Laia Riera Negre ${ }^{3}$ \\ Francisca Negre Benassar ${ }^{4}$
}

\begin{abstract}
RESUMEN
Partiendo de las necesidades educativas que presentan en el campo de las matemáticas los afectados por enfermedades raras, se plantea el diseño una estrategia didáctica centrada en potenciar el pensamiento computacional de 3 participantes de Educación Secundaria a cargo de la asociación INèDITHOS. De forma paralela, utilizando como foco el modelo de gestión de proyectos IPECC y partiendo de las necesidades en competencias digitales que muestra el equipo de voluntariado, se diseña e implementa un espacio virtual de formación centrado en el uso de los recursos que componen la misma estrategia didáctica. De esta forma, se garantiza su correcta implementación en el contexto de la asociación, así como ejemplos de sesiones, videotutoriales, recursos visuales, etc.
\end{abstract}

PALABRAS CLAVE: Enfermedades raras. Robótica educativa. Pensamiento computacional. Necesidades educativas especiales. Entorno virtual.

\footnotetext{
1 Doctor en Ciencias de la Educación. Universidad de las Islas Baleares, Palma, Baleares, España. Orcid: https://orcid.org/0000-0002-4627-2082.E-mail: s.verger@uib.es.

2 Graduado en Educación Infantil. Universidad de las Islas Baleares, Palma, Baleares, España. Orcid: https://orcid.org/0000-0001-6618-4867.E-mail: marqwhat@gmail.com.

${ }^{3}$ Graduated in Psychology. University of the Balearic Islands, Palma, Balearic Islands, Spain. Orcid: https://orcid.org/0000-0002-4766-6163. E-mail: laia.riera@uib.cat.

4 Doctor en Ciencias de la Educación. Universidad de las Islas Baleares, Palma, Baleares, España. Orcid: https://orcid.org/0000-0003-4636-2675. E-mail: xisca.negre@ uib.es.
} 
Design of a didactic strategy of computational thinking for young people with Rare Diseases of the INèDITHOS association

\begin{abstract}
Based on the educational needs of those affected by rare diseases in the field of mathematics, the design of a didactic strategy focused on enhancing the computational thinking of 3 Secondary Education participants in charge of the INèDITHOS association is proposed. In parallel, using the IPECC project management model as a focus and starting from the needs in digital skills that the volunteer team shows, a virtual training space is designed and implemented focused on the use of the resources that make up the same strategy didactic. In this way, its correct implementation is guaranteed in the context of the association, as well as examples of sessions, video tutorials, visual resources, etc.
\end{abstract}

KEYWORDS: Rare diseases. Educational robotics. Computational thinking. Special educational needs. Virtual environment.

Desenho de uma estratégia didática de pensamento computacional para jovens com Doenças Raras da associação INèDITHOS

\title{
RESUMO
}

Com base nas necessidades educacionais das pessoas afetadas por doenças raras no campo da matemática, propõe-se o desenho de uma estratégia didática voltada para o aprimoramento do pensamento computacional de 3 participantes do ensino médio responsáveis pela associação INèDITHOS. Paralelamente, usando o modelo de gerenciamento de projetos do IPECC como foco e partindo das necessidades de habilidades digitais mostradas pela equipe de voluntários, um espaço de treinamento virtual é projetado e implementado focado no uso dos recursos que compõem a mesma estratégia didático. Dessa forma, sua correta implementação é garantida no contexto da associação, bem como exemplos de sessões, tutoriais em vídeo, recursos visuais etc.

PALAVRAS-CHAVE: Doenças raras. Robótica educacional. Pensamento computacional. Necessidades educacionais especiais. Ambiente virtual. 


\section{Introducción}

Las Enfermedades Raras (EERR) se concentran en un sector muy reducido de la población, por tanto, la realidad educativa que presentan los afectados es difícilmente abordada y planteada por las administraciones.

Según Aguirre (2008), las necesidades educativas que normalmente comparten esta clase alumnado son aquellas relacionadas con: autonomía personal, limitaciones motóricas sensoriales, accesibilidad al medio físico y social, comunicación y el lenguaje, rehabilitación psicomotora, construcción de la autoimagen y finalmente aquellas que afectan al desarrollo de las capacidades cognitivas.

En referencia al contexto escolar, Monzón et al. (2017) concluyeron que las asignaturas que exigen un esfuerzo intelectual o físico importante como las matemáticas y la educación física son, generalmente, las materias más difíciles de seguir para los alumnos con alguna EERR.

Los procesos cognitivos que se llevan a cabo durante el razonamiento matemático son equivalentes a aquellos que forman parte del campo de la programación. Los alumnos parten de un reto a superar, el cual supone un proceso de reflexión, ensayo-error y análisis. Estos procesos forma parte del pensamiento computacional (PC).

El PC es una disciplina que engloba el conjunto de procesos de pensamiento que se ven envueltos en la formulación de un problema y en la expresión de su solución, de forma que esta pueda ser llevada a cabo de forma eficiente y correcta, contribuyendo al desarrollo del pensamiento abstracto, de ahí que esta clase de pensamiento en los estudiantes se convierta en pieza clave para asumir retos que implica la educación en el siglo XXI (WING, 2006, p.33).

La capacidad del alumnado para pensar de forma computacional será la encargada de seleccionar las opciones correctas y pasos a seguir con el fin de superar el reto que se le presenta. Así pues, el PC adquiere cada vez más importancia en una sociedad en constante evolución 
tecnológica, donde la escuela debe adaptar sus métodos de enseñanza a las nuevas demandas laborales y sociales.

En la actualidad, dentro del ámbito educativo, la disciplina tecnológica centrada en el uso de hardware más conocida para el tratamiento del PC es la robótica educativa.

La robótica educativa es un enfoque didáctico que puede ser fácilmente integrado en diferentes entornos educativos, en el cual mediante la utilización de dispositivos robóticos programables, y la aplicación de metodologías del aprendizaje basado en proyectos, pueden mejorar el proceso de adquisición de conocimientos de los estudiantes, permitiendo a la vez familiarizarse con las TIC y utilizarlas para definir un plan, organizar y buscar una solución a un problema específico a través de la investigación y experimentación (MISIRLI; KOMIS, 2014, p.99).

El profesorado necesita información sobre las EERR y sobre este aspecto profundizan Alfaro y Negre (2019), quienes llevaron a cabo un análisis de necesidades de información que presentan los docentes respecto a la atención educativa del alumnado con enfermedades raras en centros de Educación Infantil y Primaria de las Islas Baleares. En base al resultado del análisis, los profesores participantes consideran que serían capaces de mejorar las competencias curriculares mediante intervenciones educativas, lo que indica cierta confianza en ellos mismos, en los otros docentes y en los propios alumnos con EERR para mejorar sus habilidades y competencias. Aún así, reconocen que la formación que disponen no es suficiente confirmando la necesidad de dar a conocer las necesidades educativas del alumnado con EERR.

El contexto seleccionado para la investigación es la asociación INèDITHOS ${ }^{5}$, la cual presta el servicio de voluntariado en Mallorca (España). INèDITHOS (Investigación e Intervención para la Inclusión Educativa y Tecnológica en Pedagogía Hospitalaria) es un proyecto de voluntariado universitario dirigido a ofrecer atención psicopedagógica a niños y jóvenes que padecen enfermedades graves, en general, crónicas,

\footnotetext{
${ }^{5}$ Página web de INèDITHOS: http://inedithos.org.
} 
y, de una manera muy especial, enfermedades conocidas como enfermedades raras (EERR), minoritarias o poco frecuentes (Verger y Negre, 2019). Por otra parte, pretende ser un espacio abierto a la colaboración con investigadores para sensibilizar sobre las necesidades de estos niños y jóvenes. La asociación trabaja en tres líneas de intervención: Atención psicopedagógica en la Unidad Pediátrica de Semicríticos del Hospital Son Espases, Unidad de Curas Paliativas Pediátricas del Hospital Son Espases y finalmente, Apoyo Educativo Domiciliario, en la cual se ubicará la investigación.

A través de la metodologia aprendizaje-servicio (ApS), INèDITHOS pretende combinar procesos de aprendizaje y de servicio a la comunidad en un solo proyecto en el que los participantes se forman al trabajar sobre necesidades reales del entorno con la intención de mejorarlo.

El objetivo que se plantea para la investigación es el de identificar las características que debe reunir una estrategia didáctica para potenciar el PC en niños y jóvenes con enfermedades raras que reciben apoyo de la asociación INèDITHOS.

Este proyecto se enmarca en el proyecto de investigación EDU201679402-R (MINECO/ACI/FEDER/EU) "Soluciones educativas para la mejora de la calidad de vida del niño con enfermedad minoritaria desde una intervención innovadora y transdisciplinar" y forma parte de una acción del proyecto "INèDITnet: Xarxa per a la implementació de la metodologia Aprenentatge-Servei (ApS) en el camp de la Pedagogia Hospitalària en la Formació Universitària" de l'Institut de Recerca i Innovació Educativa (IRIE) de la Universitat de les Illes Balears (UIB).

\section{Método}

La investigación tendrá un enfoque mixto debido a que requiere del uso de técnicas situadas tanto en el marco cualitativo como cuantitativo. Al tratarse de un contexto minoritario y, con la intención de crear un soporte 
teórico que pueda ser de utilidad en situaciones similares, se llevará a cabo mediante una investigación basada en diseño (IBD) cimentada en diferentes estudios de caso. El enfoque de estudio de casos, según Ceballos-Herrera (2009), pertenece al paradigma cualitativo interpretativo, el cual considera que la realidad se construye por las personas involucradas en la situación que se estudia, y aunque el contexto de cada uno sea personal, las construcciones tienen puntos en común.

Dentro de esta metodología se ha decidido hacer uso del modelo de gestión de proyectos IPECC (Iniciar, Planificar, Ejecutar, Controlar y Cerrar) como método de implementación (Lynch y Roecker, 2007). Este modelo, ofrece la ventaja de permitir, gracias a las posibilidades iterativas que posibilita, una reevaluación continua de los resultados para garantizar un resultado de calidad, permitiendo la introducción de cambios en función de los datos obtenidos, así como mejoras.

El papel del investigador será el de diseñar uma estratégia didáctica formada por un entorno virtual para favorecer las competencias digitales del equipo de voluntariado y propuestas educativas para cada uno de los participantes con la intención de potenciar su PC, adaptándose a sus necesidades y características.

\section{Selección de casos}

El proyecto se llevará a cabo en el marco de diferentes estudios de caso con un $\mathrm{N}=3$, cada uno de ellos con edades correspondientes a la etapa de Educación Secundaria. Además, ninguno presenta el mismo diagnóstico por lo que se deberán atender de forma personalizada sus necesidades educativas y características propias.A continuación, se presentarán de forma breve a cada uno de los jóvenes. Por motivos de anonimato se identificarán con un código y no con su nombre completo. La información de cada una de las patologías ha sido extraída del listado 
de enfermedades ${ }^{6}$ que ofrece la Federación Española de Enfermedades Raras (FEDER), la cual tiene como objetivo visibilizar a las personas que padecen alguna enfermedad rara.

- I.(13 años). Distrofia muscular de Duchenne. es un tipo de distrofia muscular severo y progresivo. Los rasgos característicos son debilidad muscular y pérdida de masa muscular del cuerpo y corazón. La capacidad de caminar puede perderse sobre los 12 años. En algunos casos hay problemas de aprendizaje y memoria, así como de comunicación.

- J.(14 años). Enfermedad de Coats y parálisis. Es una enfermedad caracterizada por un desarrollo anormal de los vasos sanguíneos de la retina. Presenta pérdida de visión, desviación del alineamiento de un ojo en relación al otro y la aparición de un reflejo o mancha blanca en la pupila.

- Is.(16 años). Dolor crónico (Sin diagnóstico).

Respecto a la falta de diagnóstico que presenta uno de los participantes cabe señalar que se trata de una problemática muy habitual dentro del campo de las EERR. El retraso en el diagnóstico supone que las diferentes necesidades que presentan estas personas no pueden cubrirse hasta no tener una hoja de ruta concreta. Por tanto, cuanto menos se tarde en diagnosticar un caso, menos se tardará en adoptar las medidas necesarias para mejorar sus calidades de vida.

\footnotetext{
${ }^{6}$ Listado de enfermedades raras (FEDER): https://bit.ly/2MO58WJ.
} 


\section{Fases de la investigación}

FIGURA 1: Fases IPECC.

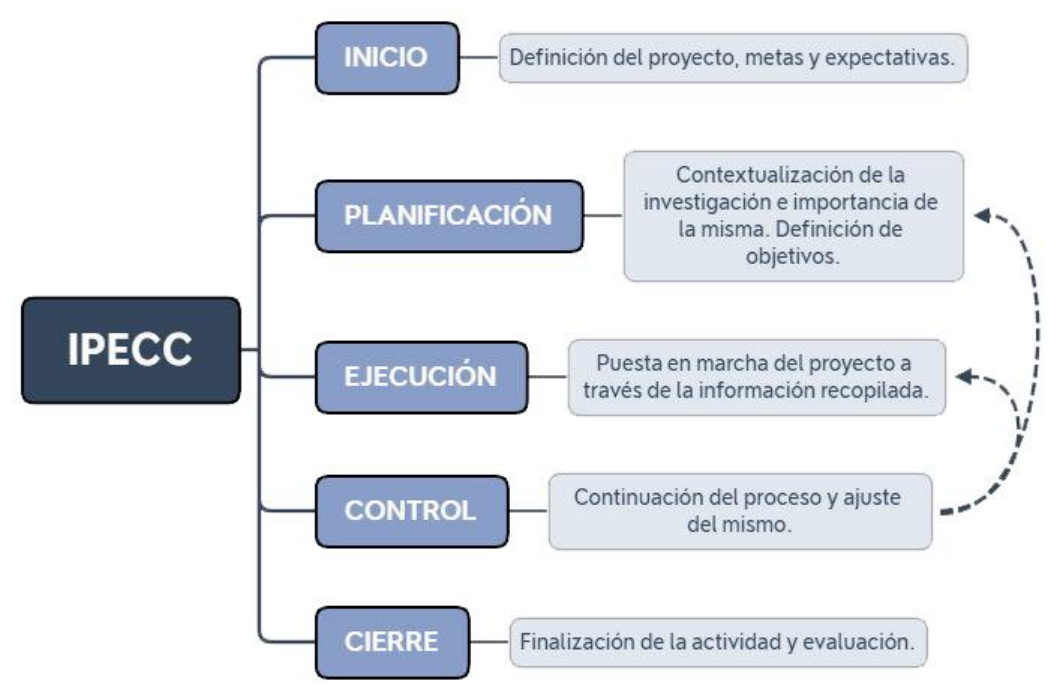

Fuente: Elaboración propia a parte de Lynch y Roecker (2007).

La investigación se va a llevar a cabo mediante el modelo de gestión de proyectos conocido como IPECC (Iniciar, Planificar, Ejecutar, Controlar y Cerrar), continuando con el proceso de investigación enmarcado en un proyecto de investigación basado en diseño.

El modelo IPECC permite repetir los ciclos necesarios hasta superar el control de calidad y poder pasar a la fase de cierre. Así pues, partiendo de el esquema presentado anteriormente se procede a explicar cada una de las fases del proyecto:

1. Fase de inicio. Se articula la visión del proyecto, se establecen metas y se definen las expectativas, así como el ámbito de aplicación. En este caso, el eje central es diseñar una propuesta enfocada a potenciar el PC de los jóvenes de INèDITHOS a través de la programación.

2. Fase de planificación. Se analiza el alcance a la vez que se identifican las tareas específicas y actividades a completar, se desarrolla un plan de proyecto y calendario. El alcance del objetivo de diseñar una estrategia didáctica para potenciar el PC abarca a 3 
usuarios de INèDITHOS y a cada una de las voluntarias que acuden semanalmente a sus domicilios. Por otra parte, el objetivo de diseñar e implementar una plataforma virtual de formación engloba a todas las voluntarias de la asociación para que todas puedan acceder y desarrollar sus competencias digitales.

3. Fase de ejecución. En esta fase se analizan los resultados obtenidos sobre las competencias de las voluntarias y, por tanto, basándose en sus necesidades, se empieza a diseñar el esquema de la plataforma virtual para que su implementación en Moodle sea más ágil. Por otra parte, teniendo a disposición datos sobre los participantes se empieza a hacer la selección de recursos incluidos en la futura estrategia didáctica, en base a sus necesidades y características.

4. Fase de control. Durante esta fase se controlará el diseño e implementación del entorno virtual de formación. Mientras tanto, las voluntarias de la asociación interactuarán con el entorno y realizarán una evaluación. A su vez, se procederá a la validación de un cuestionario para adjuntar en el entorno virtual con la intención de que sirva como herramienta a futuro para valorar posibles propuestas basadas en PC que surjan desde la propia asociación.

5. Fase de cierre. Una vez terminado el entorno virtual de formación se elaborán las conclusiones en las cuales se detallarán todos los aspectos clave que se han tenido en cuenta para el correcto diseño e implementación del entorno de formación virtual así como del diseño de la estrategia didáctica basada en PC.

\section{Instrumentos utilizados}

A continuación, se expondrán los instrumentos usados para la obtención y análisis de información, explicando a su vez las adaptaciones necesarias que se han realizado para su aplicación en el contexto de voluntariado de la 
asociación. Para poder llegar al equipo de voluntariado, los cuestionarios han sido enviados a través de la plataforma Formularios de Google.

\section{Cuestionario de percepción del profesorado de Educación Primaria sobre el aprendizaje del alumnado basado en competencias (Meroño, L. et al., 2016).}

Ante la necesidad de conocer las capacidades actuales de los jóvenes a los que irá dirigida la estratégia didáctica, el instrumento selecionado permite recopilar información sobre sus competencias generales mediante una escala Liker del 1 al 5. Para ello, se han debido adaptar los ítems a otros equivalentes a la etapa de Educación Secundaria. De forma complementaria a la adaptación de ítems, y con el fin de que las sesiones sean lo más personalizadas en cada caso, se añadieron 11 preguntas abiertas para conocer la personalidad del participante, aficiones, dispositivos electrónicos con los que cuenta, entre otros aspectos.

\section{Proceso de adaptación del cuestionario a Educación Secundaria}

Para la adaptación del cuestionario a Educación Secundaria se han seleccionado indicadores correspondientes a la etapa educativa en el Decreto 34/2015, del 15 de mayo, en el cual se establece el currículo de la educación secundaria obligatoria en las Islas Baleares y, por otra parte, en el Orden ECD/65/2015, de 21 de enero, por la que se describen las relaciones entre las competencias, los contenidos y los criterios de evaluación de la educación primaria, educación secundaria obligatoria y el bachillerato. 
TABLA 2: Ejemplo del proceso de adaptación 7 a Educación Secundaria de los ítems del Cuestionario de percepción del profesorado de Educación Primaria basado en competencias (MEROÑO, L. et al., 2016).

\begin{tabular}{|l|l|l|}
\hline Ítems cuestionario original & Justificación del cambio & \multicolumn{2}{|c|}{$\begin{array}{c}\text { Ítems adaptados a } \\
\text { Secundaria }\end{array}$} \\
\hline $\begin{array}{l}\text { 1. Es capaz y quiere } \\
\text { conocer las partes y } \\
\text { funciones del cuerpo } \\
\text { humano, por ejemplo, los } \\
\text { aparatos y sistemas. }\end{array}$ & $\begin{array}{l}\text { El ítem es el mismo solo que } \\
\text { redactado tal y como aparece } \\
\text { en el Currículum de Educación } \\
\text { Secundaria. }\end{array}$ & $\begin{array}{l}\text { componentes del aparato } \\
\text { digestivo, circulatorio, } \\
\text { respiratorio y excretor, } \\
\text { conociendo ar and } \\
\text { funcionamiento. }\end{array}$ \\
\hline
\end{tabular}

Fuente: Elaboración propia.

\section{Cuestionario de competencias en TIC para profesores de distintos} niveles educativos (de la IGLESIA, J. et al., 2016).

A la hora de establecer los contenidos a incluir en el espacio virtual cabe tener en cuenta las necesidades de formación de las voluntarias de la asociación. Debido a que gran parte de las voluntarias cursan el Grado Educación Primaria y Educación Infantil y/o profesoras en activo, el cuestionario seleccionado engloba todas las etapas educativas en un mismo instrumento. El cuestionario está compuesto por 45 indicadores evaluables mediante una escala Likert del 1 al 5, separados en tres grandes grupos: uso técnico (13), uso didáctico (16) y diseño de medios (16).

\section{Cuestionario de evaluación de la calidad de un sistema de gestión de aprendizaje (LÓPEZ, I. L. et al., 2018).}

Para ofrecer un espacio virtual que se adapte a las necesidades formativas de las voluntarias es necesario conocer su grado de satisfacción con la plataforma una vez sea implementada, con el fin de realizar las modificaciones pertinentes y conseguir que el entorno incentive un uso significativo. El cuestionario originalmente cuenta con 33 preguntas categorizadas en tres apartados diferentes: Frecuencia de uso del sistema,

\footnotetext{
${ }^{7}$ Proceso de adaptación completo del cuestionario a Educación Secundaria: https://bit.ly/3fkXYG8.
} 
calidad del sistema y evaluación global del sistema. Para su aplicación ${ }^{8}$ a las voluntarias de la asociación INèDITHOS se ha modificado la terminologia de dos preguntas y, por otra parte, se han suprimido cuatro preguntas que interpretaban el espacio virtual como una asignatura a cursar, y por tanto, inservible para el caso en cuestión.

\section{Recursos de robótica educativa utilizados}

Los recursos que formarán parte de la estrategia didáctica y el espacio virtual de formación se han seleccionado entre aquellos que son accesibles para la asociación o ya son parte de su propiedad, entre los que se encuentran: Scratch, mBot, Sphero ball mini, La hora del código, Edison, Lego BOOST, Makey Makey y Megamaker Kit.

\section{Resultados y discusión}

Para el diseño de la estratégia didáctica se recopilaron los resultados que cada voluntaria otorgó sobre el joven al que realiza el voluntariado semanalmente en su domicilio a través del Cuestionario de percepción del profesorado de Educación Primaria sobre el aprendizaje del alumnado basado en competencias (Meroñol et al., 2016), adaptado a Educación Secundaria.

na vez asimiladas las competencias generales, características ${ }^{9} \mathrm{y}$ aficiones de los tres jóvenes se presenta la propuesta didáctica para cada uno de ellos. Cada propuesta está formada por cinco sesiones diseñadas para poder llevarse a cabo con una temporalidad cercana a los cuarenta minutos, adecuándose a la duración normativa de la actividad de voluntariado (hora y media) y a los dispositivos electrónicos que dispone el joven.

\footnotetext{
${ }^{8}$ Modificaciones implementadas en el Cuestionario de evaluación de la calidad de un sistema de gestión de aprendizaje (LÓPEZ, I. L. et al., 2018): https://bit.ly/3fnYPpw.

${ }^{9}$ Resultados de las preguntas abiertas del Cuestionario de percepción del profesorado de Educación Primaria sobre el aprendizaje del alumnado basado en competencias (MEROÑOL, L. et al., 2016): https://bit.ly/2ZZz1tq.
} 
TABLA 5: Resultados de $\quad$ - I. $\left(13\right.$ años). ${ }^{10}$ La primera sesión cada participante.

Programamos una historia, introducirá el

\begin{tabular}{|c|c|c|c|}
\hline $\begin{array}{c}\text { Indicador } \\
\text { del } \\
\text { cuestionario }\end{array}$ & 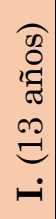 & 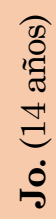 & 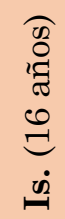 \\
\hline 1 & 2 & 4 & 4 \\
\hline 2 & 2 & 2 & 5 \\
\hline 3 & 2 & 4 & $\sim$ \\
\hline 4 & 3 & 1 & 5 \\
\hline 5 & 4 & 4 & 5 \\
\hline 6 & 2 & 2 & 4 \\
\hline 7 & 1 & 2 & 4 \\
\hline 8 & 1 & 2 & 3 \\
\hline 9 & 1 & 3 & 4 \\
\hline 10 & 1 & 1 & 4 \\
\hline 11 & 2 & 1 & 4 \\
\hline 12 & 1 & 2 & 4 \\
\hline 13 & 1 & 2 & 4 \\
\hline 14 & 1 & 3 & 4 \\
\hline 15 & 2 & 1 & 3 \\
\hline 16 & 3 & 4 & 4 \\
\hline 17 & 1 & 3 & 4 \\
\hline 18 & 2 & 2 & 4 \\
\hline 19 & 3 & 4 & 5 \\
\hline 20 & 3 & 4 & 5 \\
\hline 21 & 3 & 4 & \\
\hline 22 & 2 & 3 & 3 \\
\hline 23 & 2 & 3 & 3 \\
\hline 24 & 1 & 2 & 4 \\
\hline
\end{tabular}
entorno Scratch y algunos de los bloques que lo componen mediante un tutorial ofrecido por la propia plataforma para crear personajes $\mathrm{y}$ moverlos. En la segunda sesión Creamos un videojuego, mediante un videotutorial incorporado en el espacio virtual de formación diseñado, el joven deberá seguir las directrices expuestas, asimilando así los angulos $\mathrm{X}$ e $\mathrm{Y}$ mediante Scratch. En la tercera sesión No te salgas de la línea, se incorpora el robot mBot para mostrar su funcionamiento a través de un recorrido creado con cinta aislante en el suelo, teniendo como objetivo programar el siguelíneas para que el robot no se salga de la cinta. En la cuarta sesión No todos los robots tienen la misma forma, gracias a Sphero Ball, pondrá a prueba sus conocimientos sobre programación por bloques para sortear obstáculos mediante el robot. Como última sesión, en Cómo se hizo Minecraft, aprovechando su afición por lo videojuegos utilizará la plataforma La hora del código para realizar la lección 19 del curso rápido y conocer los aspectos internos de programación del videojuego Minecraft de forma lúdica.

- Jo. (14 años). ${ }^{11}$ En la primera sesión El brazo mecánico, gracias a las piezas de Lego BOOST construirá y programará un pequeño brazo mecánico que luego utilizará para comprobar si resiste el peso de algunos objetos de su domicilio. En la segunda sesión realizará al igual que el participante anterior

\footnotetext{
${ }^{10}$ Guía detallada de la propuesta didáctica de $I$ : https://bit.ly/2ZjNbGA.

${ }^{11}$ Guía detallada de la propuesta didáctica de $J o$ : https://bit.ly/2OjCMoc.
} 
la propuesta No todos los robots tienen la misma forma. La tercera sesión Esquiva los obstáculos permitirá a Jo. crear un recorrido en el suelo con objetos de su entorno para sortear los obstáculos programando el robot Edison. En la cuarta sesión Controlando el tráfico se utilizarán algunos componentes de Megamaker Kit para crear y programar un semáforo, siguiendo una serie de instrucciones propuestas en la web Ebotics. Finalmente, en la última sesión Aprendiendo a conducir combinará las dos últimas actividades programando a Edison para que se detenga cuando el semáforo esté en rojo y avance cuando esté verde.

- Is. (16 años). ${ }^{12} \mathrm{Al}$ ser una persona muy curiosa frente a situaciones nuevas y con ganas de aprender se ha procurado una gran variedad de recursos. En la primera sesión Creamos un videojuego Is. creará mediante Scratch un videojuego basado en el popular juego Pong, siguiendo una serie de directrices en un videotutorial incluido en el espacio virtual de formación. El videojuego podrá ser controlado con Makey Makey conectado a cualquier objeto cotidiano. La segunda sesión será El brazo mecánico (Lego BOOST), explicada anteriormente, al igual que la tercera será No todos los robots tienen la misma forma (Sphero Ball Mini). La cuarta sesión será No te salgas de la línea, correspondiente al recurso mBot, el cual deberá ser programado para no salirse del recorrido de cinta aislante. Para finalizar la propuesta, Is. creará y programará al igual que Jo. un semáforo, confluyendo así habilidades motrices y habilidades cognitivas.

Para el diseño del espacio virtual de formación centrado en el uso de recursos de $\mathrm{PC}$ se utilizaron los resultados que las 16 voluntarias mostraron en las tres dimensiones del Cuestionario de competencias en TIC para profesores de distintos niveles educativos (de la Iglesia, Fernández y Cebreriro, 2016).

\footnotetext{
${ }^{12}$ Guía detallada de la propuesta didáctica de $I s$ : https://bit.ly/3gJA9Z0.
} 
GRÁFICA 1: Resultados de la dimensión "Uso técnico de las TIC".

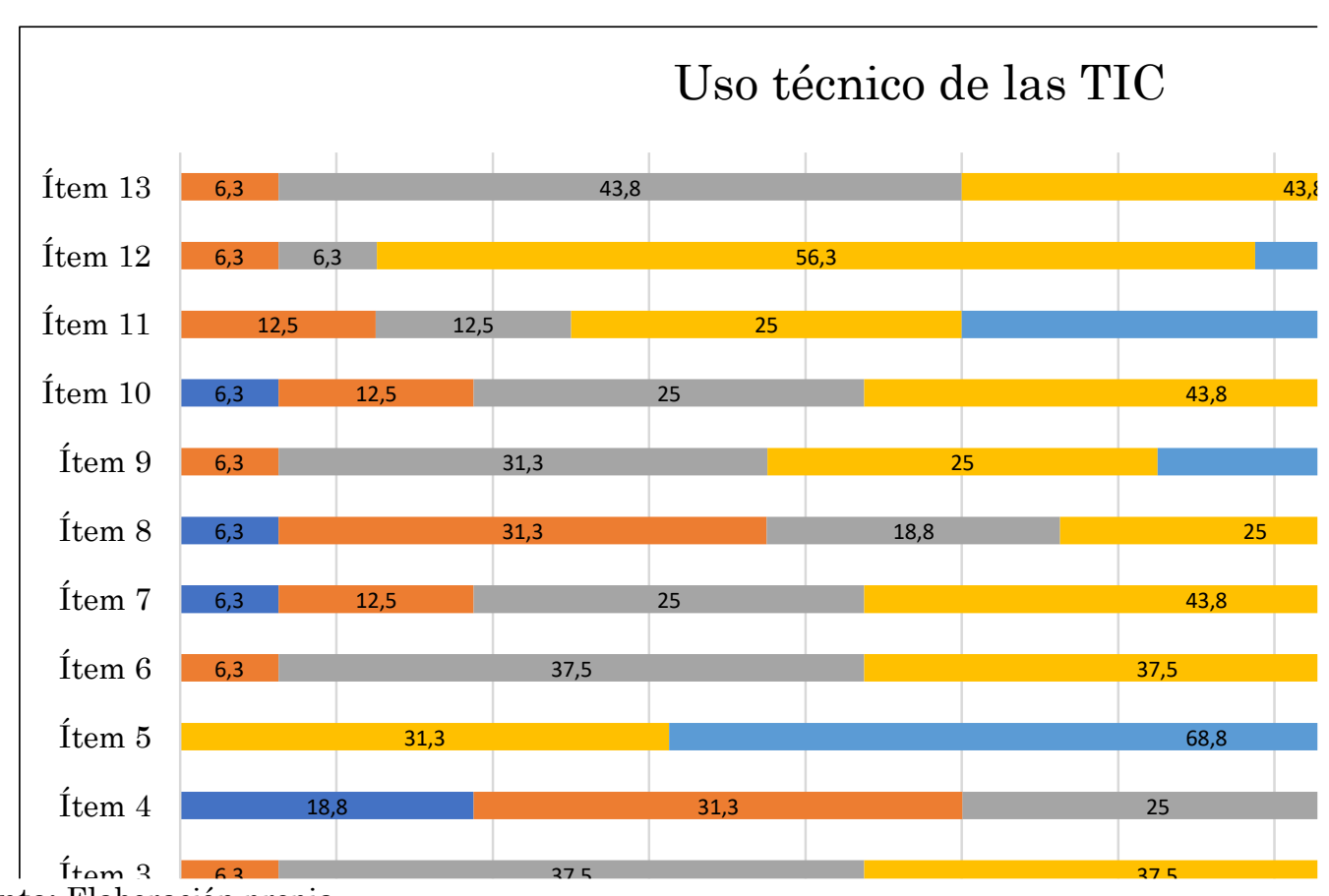

Fuente: Elaboración propia.

GRÁFICA 2: Resultados de la dimensión "Uso didáctico de las TIC".

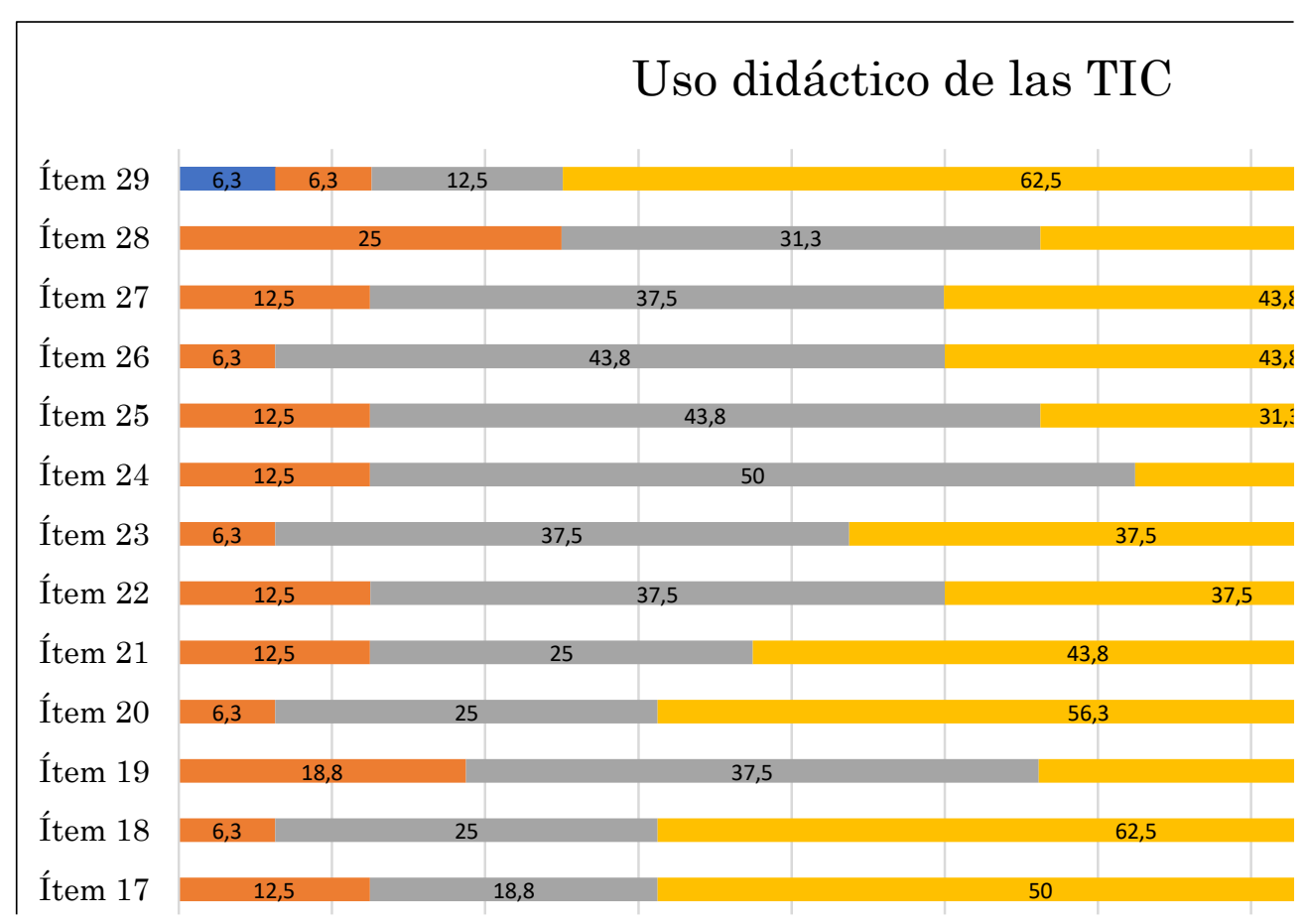

Fuente: Elaboración propia. 
GRÁFICA 3: Resultados de la dimensión "Diseño de medios mediante TIC".

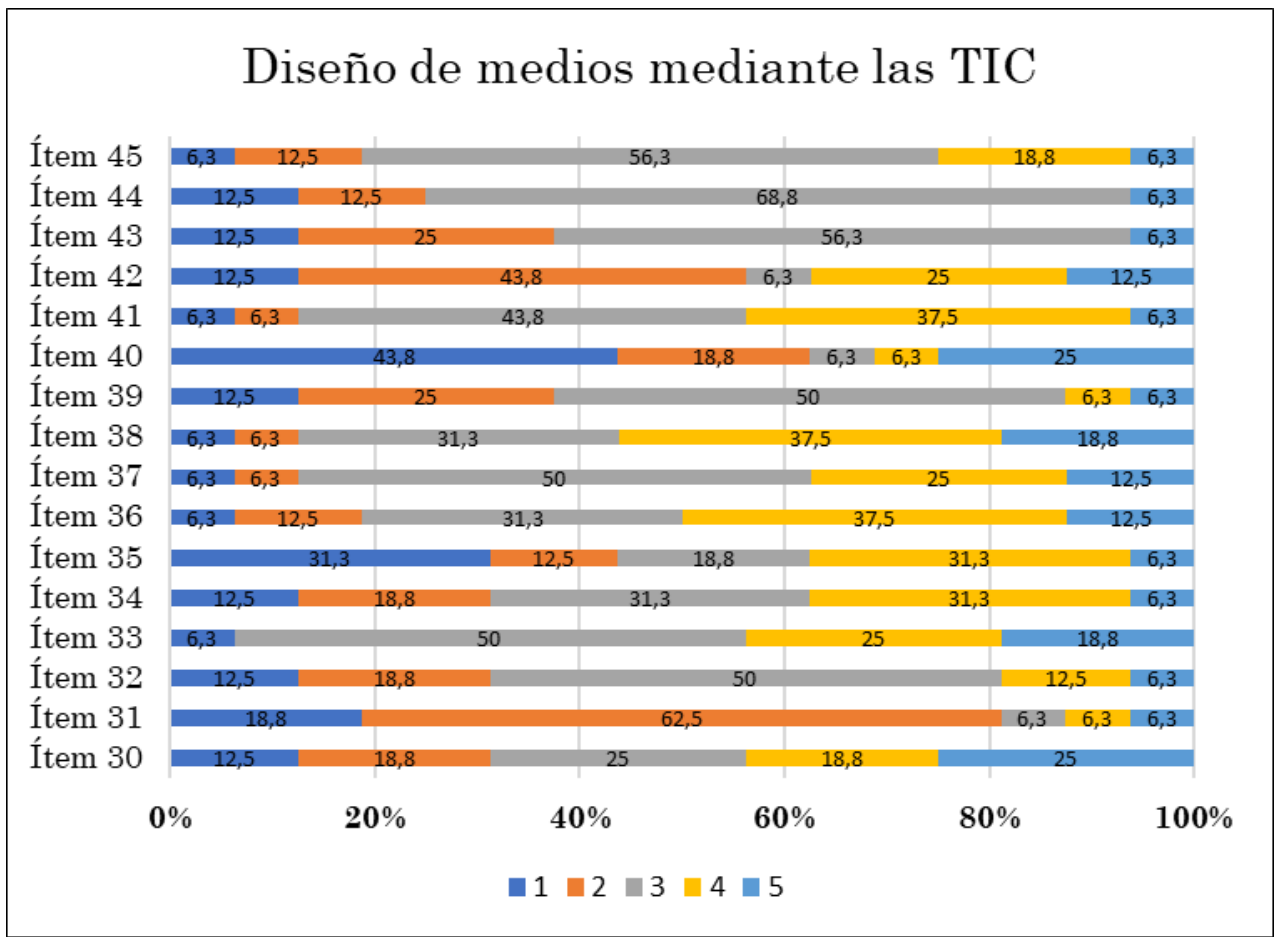

Fuente: Elaboración propia.

Una vez recopilados los datos del primer apartado se observa como en la totalidad de los ítems hay mínimo una voluntaria que siente que no es nada competente respecto al uso de las TIC. De igual forma, todas las preguntas tienen un mayor porcentaje de respuestas superiores a la puntuación media (3), por lo que será positivo de cara a la implementación del espacio virtual. En los indicadores de la segunda gráfica se distingue una tendencia más regular que en el anterior caso. De forma rotunda, las voluntarias en general se sienten competentes en el uso de las TIC con fines didácticos. De esta manera, el uso de la robótica educativa no se percibe problemático si se acompaña de la formación virtual adecuada.

Finalmente, en el tercer y último apartado que se presentará a continuación, hay una gran cantidad de ítems en los que hay dos voluntarias que no se sienten capaces, llegando en algunos casos a superar el tercio de participantes que se perciben a sí mismas como nada competentes. Como algunos de esos ítems implican el uso de software como herramienta didáctica, se tendrá en cuenta a la hora de definirlos en el espacio virtual, 
ofreciendo tutoriales y documentación que ayude a facilitar la implementación de esa clase de recursos.

El diseño y desarrollo del espacio virtual de formación se ha llevado a cabo mediante la plataforma Moodle teniendo en cuenta los resultados mostrados anteriormente. Por ello, atendiendo a las necesidades que presentan las voluntarias respecto al uso de software educativo con fines didácticos, se ha procurado que la implementación de recursos virtuales esté explicada de la forma más detallada y accesible posible.

Así pues, se procede a presentar el del espacio virtual de formación enfocado a Educación Secundaria formado por cuatro apartados (Recursos y sesiones, Espacio general, Tutoriales y Ampliación). Así pues, en la imagen se enumeran los apartados para explicarlos después.

FIGURA 2: Componentes del bloque "Recursos y sesiones".

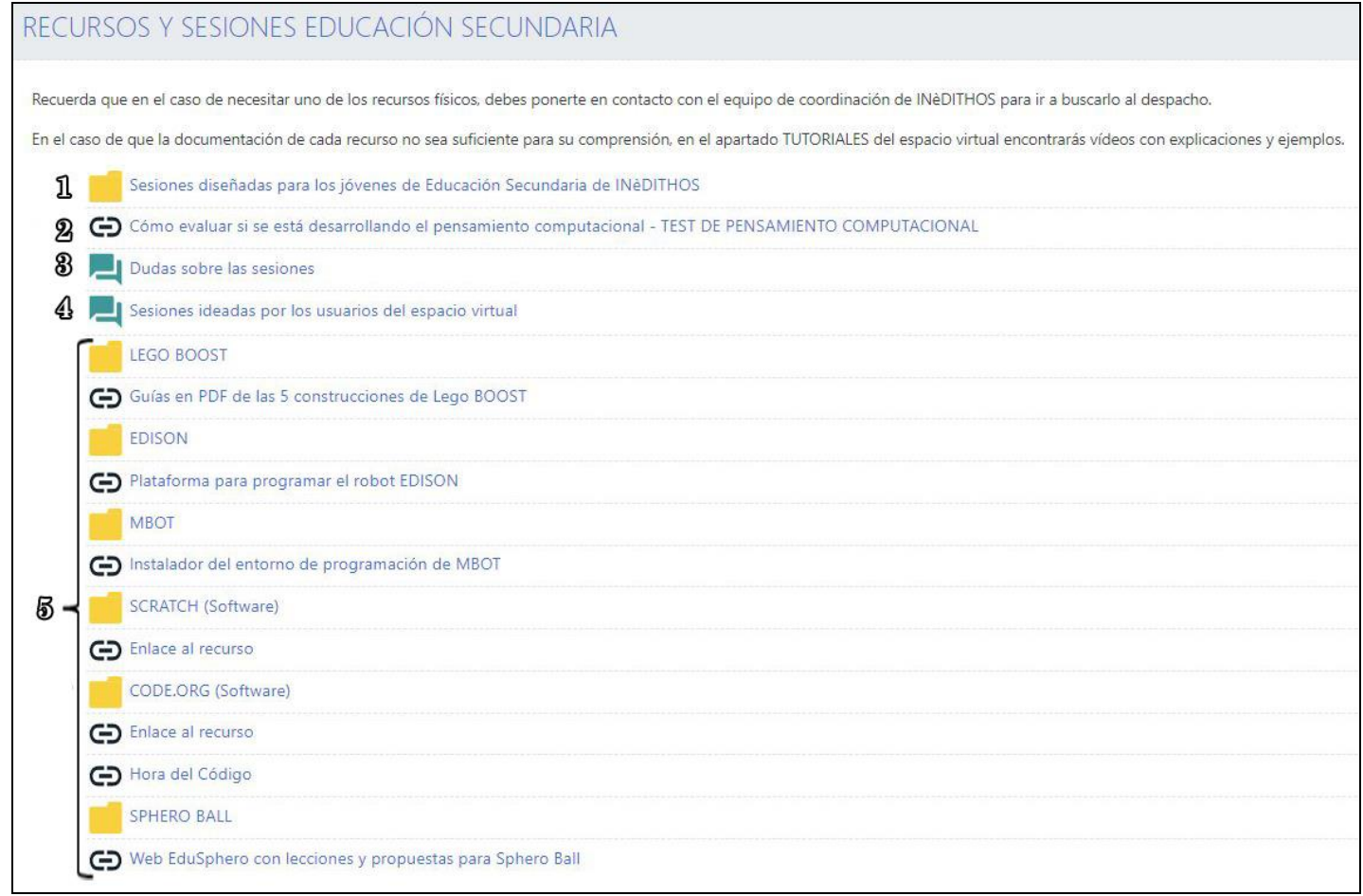

Fuente: Elaboración propia.

1. Sesiones diseñadas para los jóvenes de Educación Secundaria. En este apartado se incluyen las guías detalladas de las sesiones propuestas para cada participante, de esta forma, cada voluntaria 
podrá conocer los recursos que necesitará para las actividades y los objetivos que se pretenden cubrir.

2. Test de Pensamiento Computacional (Román, 2015). Cuestionario que permite conocer el nivel de PC de alumnos de Educación Secundaria en términos de preguntas y planteamientos basados en programación por bloques. Al ser implementado como Pretest y Postest las voluntarias podrán conocer cuánto ha sido potenciado el PC con la estrategia didáctica.

3. Dudas sobre las sesiones. Espacio que permite a las voluntarias de INèDITHOS realizar cualquier consulta sobre las actividades a implementar.

4. Sesiones ideadas por los usuarios del espacio virtual. Espacio habilitado para compartir experiencias basadas en PC ideadas por las propias voluntarias.

5. Lego BOOST, Edison, mBot, Scratch, Code.org y Sphero Ball. Dentro de cada una de las carpetas la voluntaria tiene a su disposición un archivo PDF con una breve descripción del material y ejemplos de sesiones con el mismo. En el caso de recursos software se han adjuntado enlaces en la página principal que facilitarán el acceso al contenido por parte de las voluntarias.

FIGURA 3: Componentes del bloque "Espacio General”.

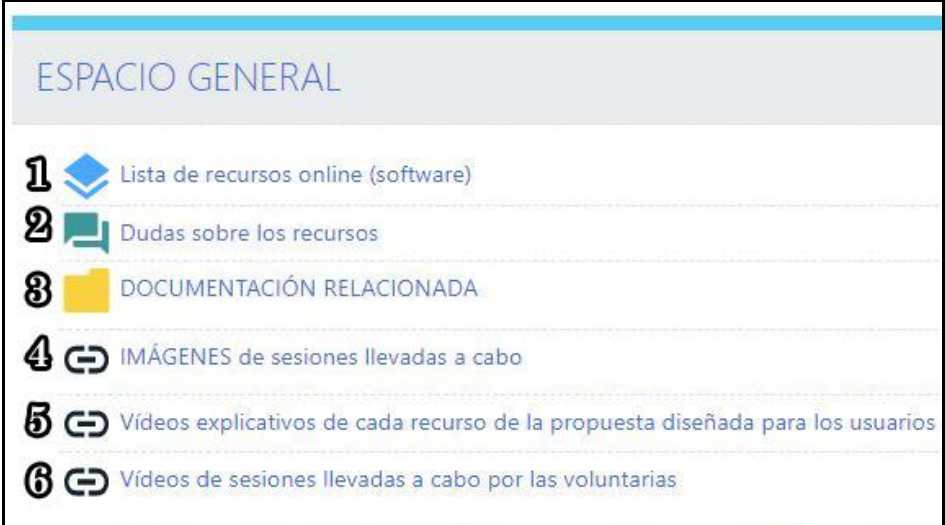

Fuente: Elaboración propia. 
El apartado Espacio General está compuesto por los siguientes elementos:

1. Lista de recursos software. Wiki colaborativa con software online basado en PC, descritos y con enlace de acceso. Como por ejemplo: Lightbot, Gamesonomy o Compute It!.

2. Dudas sobre los recursos. Foro para cuestiones relacionadas con cualquiera de los recursos que sean incluídos en la wiki de software.

3. Documentación relacionada. Lecturas relacionadas con las EERR y/o la robótica educativa en contextos similares al que presenta la asociación.

4. Imágenes de sesiones llevadas a cabo. Enlace a carpeta Drive donde compartir imágenes de las sesiones que las voluntarias pongan en práctica con los participantes.

5. Vídeos explicativos de cada recurso de la propuesta de cada usuario. Enlace a una lista de reproducción privada vinculada al canal de Youtube de la asociación con vídeos sobre los recursos que componen la estrategia didáctica.

6. Vídeos de sesiones llevadas a cabo por las voluntarias. Enlace a otra lista de reproducción donde a través de la coordinadora del canal, las voluntarias podrán adjuntar vídeos grabados por ellas mismas sobre sesiones que hayan implementado.

Como parte de la fase de la fase de control del ciclo IPECC, el espacio virtual de formación fue sometido a evaluación por parte de las 16 integrantes que componen el equipo de voluntariado de la asociación INèDITHOS, de las cuales dieron respuesta 13. Mediante la aplicación del Cuestionario para evaluar la calidad de un sistema de gestión de aprendizaje (López, I. L. et al., 2018) se obtuvieron los datos necesarios para implementar cambios que respondan a las necesidades que presenten las usuarias. 
GRÁFICA 4: Resultados de la dimensión "Frecuencia de uso del sistema".

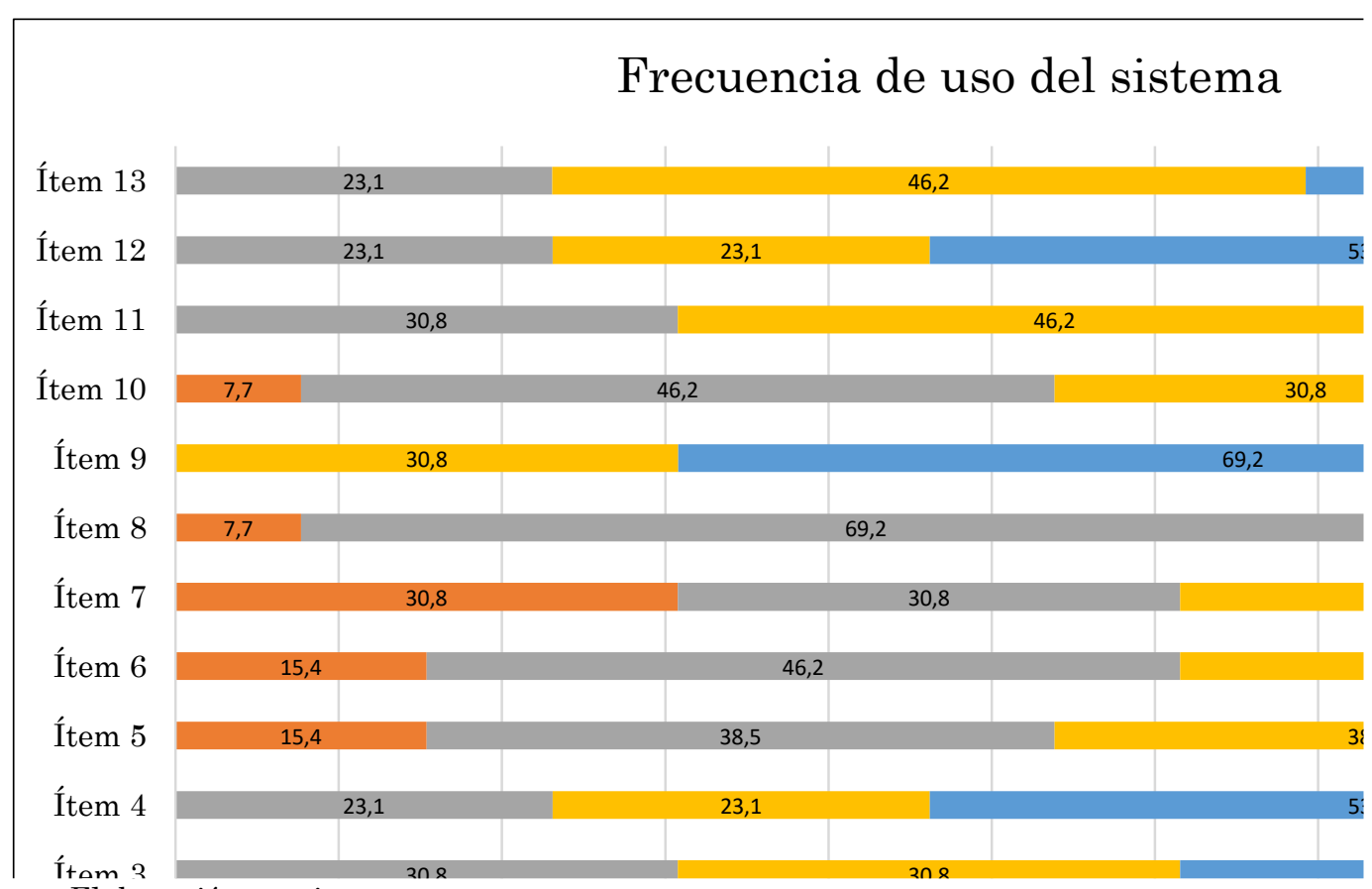

Fuente: Elaboración propia.

GRÁFICA 5: Resultados de la dimensión "Calidad del espacio virtual".

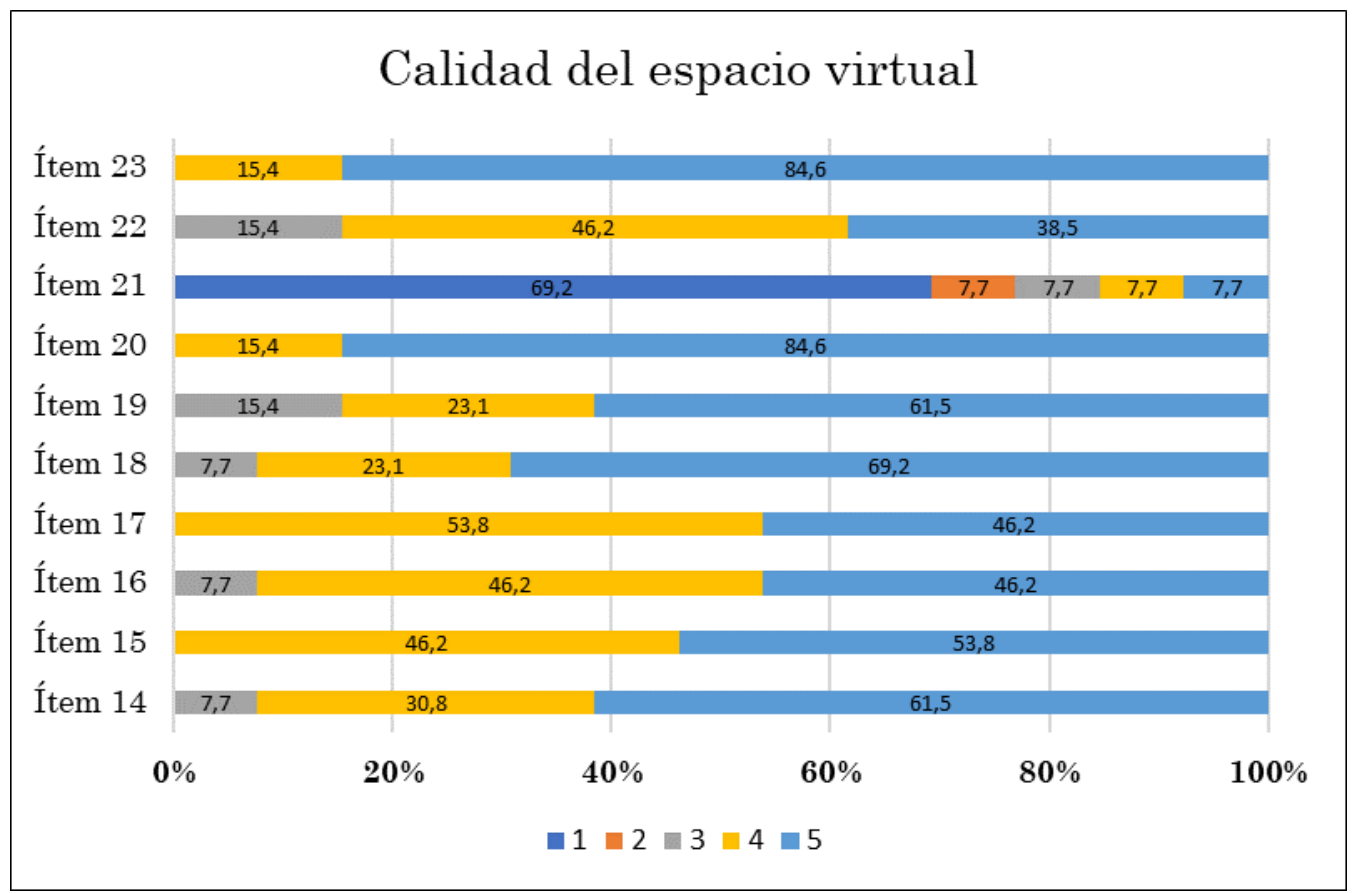

Fuente: Elaboración propia. 
GRÁFICA 6: Resultados de la dimensión "Evaluación global del sistema”.

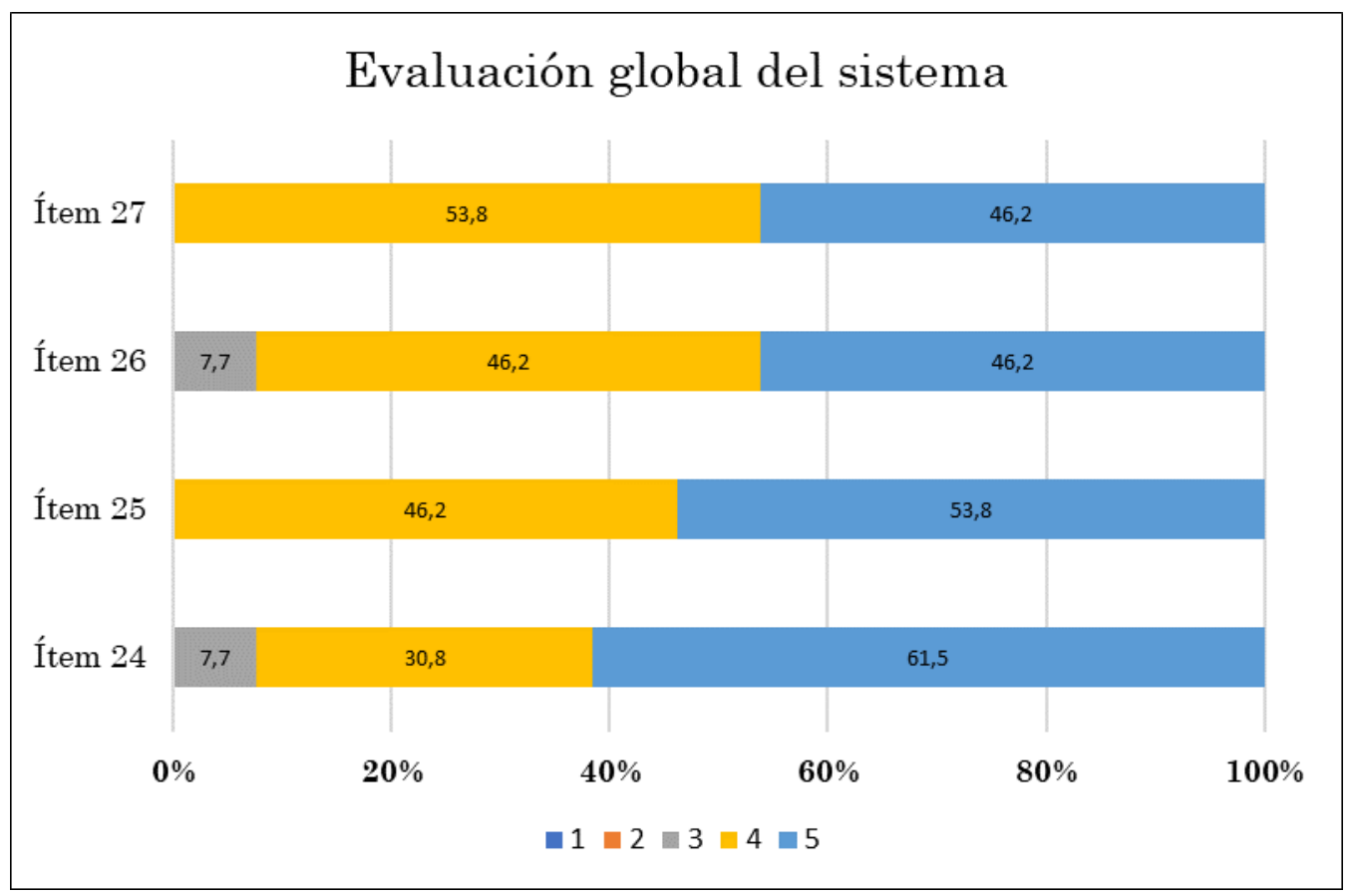

Fuente: Elaboración propia.

Los resultados muestran una valoración muy positiva en todos los aspectos, sin embargo, los indicadores que hacen referencia a la comunicación a través del entorno $(5,6$ y 7) se ven más afectados debido a que las voluntarias disponen del número telefónico de sus compañeras y tienen prioridad por utilizar sistemas de mensajería instantánea como WhatsApp. En la segunda gráfica, al igual que en la primera, se muestra cómo las voluntarias creen que disponen de un entorno de calidad. En el caso del indicador 21, ha presentado dudas debido a un error de entendimiento que las voluntarias comunicaron uma vez enviado el cuestionario, ya que no eran conscientes de que la valoración alta da a entender que la plataforma presenta problemas de navegación.

En la tercera gráfica, la valoración de las voluntarias en cuanto a calidad del espacio virtual es más que satisfactoria en los cuatro indicadores. Por tanto, teniendo en cuenta las tres dimensiones analizadas se puede afirmar que el entorno de formación obtiene una validación positiva de las usuarias. Por otra parte, los comentarios que las voluntarias adjuntaron en el cuestionario se han tenido en cuenta a la hora de modificar el espacio como parte del ciclo IPECC. Los cambios introducidos hacen referencia a errores de descarga de algunos archivos que a día de 
hoy han sido reparados, y falta de entendimiento de algunos elementos que han sido minimizados añadiendo una breve descripción visible en el menú principal.

Así pues, se da por concluido el diseño y desarrollo del entorno virtual de formación basado en PC para la asociación INèDITHOS. El cuestionario utilizado para la evaluación del espacio (López, Zapata, Briceño y Poot, 2018) se dejará de forma permanente para futuras modificaciones. Finalmente, se adjuntará un correo de contacto para cualquier problemática que pueda surgir en el espacio.

\section{Conclusión}

El presente artículo tenía como objetivo identificar las características que debe reunir una estrategia didáctica para potenciar el PC de jóvenes con EERR de la asociación INèDITHOS. En consecuencia, se han establecido una serie de aspectos a tener en cuenta a la hora de diseñar e implementar las sesiones.

Las actividades planteadas deben sustentarse en aficiones y gustos del joven al cual van dirigidas. Para ello, será indispensable que la voluntaria que lleve a cabo las actividades junto a cada uno de ellos sea la misma que va al domicilio semanalmente a prestar el servicio de voluntariado. En el caso de que fuese una persona desconocida, la falta de vínculo podría afectar al desarrollo de la propuesta por una posible incomodidad del joven.

A la hora de diseñar una propuesta, la voluntaria debe detectar las competencias generales del niño joven, así como las necesidades educativas que presente en cualquier ámbito más allá de las matemáticas. Una vez se conocen las competencias generales se deben entender las características clave de la enfermedad que padece, ya que en el momento de diseñar una propuesta didáctica habrá que tener en cuenta hasta qué punto la dolencia supone alguna limitación a nivel motriz y cognitivo.

Independientemente de si el niño o joven está escolarizado o no, se debe evitar confundir la edad biológica con la edad cognitiva. Es común encontrar ocasiones donde la diferencia entre estas dos edades es mayor a cinco años. En estos casos, la edad biológica no es más que una etiqueta, por 
lo que el grado de dificultad inicial de la estrategia didáctica se establecerá en base a sus conocimientos y no en base a la edad biológica o curso al que pertenezca. Así pues, el grado de dificultad de las sesiones debe ir siempre en aumento por lo que conviene reforzar con asiduidad conceptos trabajados con anterioridad, intentando que el niño o joven verbalice los pasos que hace durante el proceso de la actividad para una mejor interiorización.

Las sesiones deben encontrar un equilibrio entre duración y eficacia. Una actividad de duración excesiva, aunque esté formada por diferentes recursos, conceptos e intervenciones por parte del niño puede ser contraproducente por el desgaste mental que supone. Por lo tanto, se priorizará siempre una propuesta de corta duración que sea flexible a alargarse en el caso de que el interés del niño de pie a ello.

Las voluntarias deben tener a su disposición un espacio virtual al que acudir para autoformarse de cara a la elaboración y/o implementación de una estrategia didáctica. Un espacio visualmente atractivo y ordenado donde encontrará recursos que puede incluir en las propuestas e ideas de cómo implementarlos en el contexto de la asociación, de una forma clara que incentive su uso. La presentación de los recursos y actividades influyen enormemente en la predisposición a llevarlas a cabo, por lo que la voluntaria debe garantizar una atractiva introducción de la propuesta para el joven. A su vez, en caso de duda entre un recurso físico y otro virtual se dará prioridad siempre al físico (siempre y cuando el niño o joven pueda establecer contacto con él). El hecho de interactuar físicamente con el material estimula la acción y el sentido del tacto, cosa que los recursos virtuales no ofrecen.

\section{Referencias}

AGUIRRE, P et al. Enfermedades raras y crónicas. Manual de atención al alumnado con necesidades específicas de apoyo educativo por padecer enfermedades raras y crónicas. Junta de Andalucía, 2008. Disponible en: http://hdl.handle.net/11162/3178.

ALFARO, A. y NEGRE, F. Análisis de las necesidades de información que presentan los docentes respecto a la atención educativa del alumnado con enfermedades raras. Revista Electrónica Interuniversitaria de Formación del Profesorado, v. 22, n. 1, p. 175-194, 2019. DOI:

https://doi.org/10.6018/reifop.22.1.326341. 
CEBALLOS-HERRERA, F. A. El informe de investigación con estudio de casos. Magis. Revista Internacional de Investigación en Educación, v.1, n. 2, p. 413-423, 2009. Disponible en: https://bit.ly/2OgiFXM.

FERNÁNDEZ DE LA IGLESIA, J. et al. Desarrollo de un cuestionario de competencias en tic para profesores de distintos niveles educativos. PíxelBit. Revista de Medios y Educación, v. 0, n. 48, p. 135-148, 2016. DOI: http://dx.doi.org/10.12795/pixelbit.2016.i48.09.

LÓPEZ, I. L. et al. Diseño y validación de un instrumento para evaluar la calidad de un sistema de gestión de aprendizaje. Tecnologías y Aprendizaje: Investigación y Práctica, p. 411-418, 2018. Disponible en: https://bit.ly/2y78n7F.

MEROÑO, L. et al. Diseño y validación del cuestionario de percepción del profesorado de Educación Primaria sobre el aprendizaje del alumnado basado en competencias (\# ICOMpri2). Revista Complutense de Educación, v. 29, n. 1, p. 215-235, 2016. DOI: http://dx.doi.org/10.5209/RCED.52200.

MISIRLI, A., y KOMIS, V. Robotics and programming concepts in early childhood education: A conceptual framework for designin educational scenarios. Research on e-Learning and ICT in Education, v. 0, p. 99-118, 2014. DOI: https://doi.org/10.1007/978-1-4614-6501-0_8.

MONZÓN-GONZÁLEZ, J. et al. Alumnado con enfermedades poco frecuentes y escuela inclusiva. Octaedro, 2017.

LYNCH, M. M. y ROECKER, J. Project managing e-learning: A handbook for successful design, delivery and management. Routledge, 2007.

ROMÁN-GONZÁLEZ, M. et al. Test de Pensamiento Computacional: diseño y psicometría general. III Congreso Internacional sobre Aprendizaje, Innovación y Competitividad (CINAIC 2015). DOI:

https://doi.org/10.13140/RG.2.1.3056.5521.

NEGRE, F. y VERGER, S. INèDITHOS. Intervenció i investigació educativa i tecnológica en Pedagogia Hospitalaria. Bones Pràctiques en Entorns Pedagògics. Universitat de les Illes Balears, v. 3, 2019. Disponible en: https://bit.ly/2UrXsxx.

WING, J. Computational Thinking. Communications of the Acm, v. 49, n.3, p. 33-35, 2006. DOI: http://dx.doi.org/10.1098/rsta.2008.0118.

\section{Anexo}

Carpeta contenedora de Google Drive con los documentos externos adjuntados como notas a pie de página: https://bit.ly/3iY5sB2.

Recebido em abril de 2020. Aprovado em dezembro de 2020. 